\title{
Conhecimento sobre uso de fluoretos em saúde bucal coletiva entre coordenadores municipais de saúde bucal do Estado de Santa Catarina, Brasil
}

doi: 10.5123/S1679-49742012000100009

\author{
Knowledge about the use of fluoride in community dentistry among municipal oral health \\ authorities of Santa Catarina State, Brazil
}

Andreia Morales Cascaes

Programa de Pós-Graduação em Epidemiologia, Universidade Federal de Pelotas, Pelotas-RS, Brasil

Luciana Claro Berben Kamimura

Universidade Federal de Santa Catarina, Florianópolis-SC, Brasil

Karen Glazer Peres

Departamento de Saúde Pública, Universidade Federal de Santa Catarina, Florianópolis-SC, Brasil

Marco Aurélio Peres

Departamento de Saúde Pública, Universidade Federal de Santa Catarina, Florianópolis-SC, Brasil

\section{Resumo}

Objetivo: verificar o grau de conhecimento dos coordenadores municipais de Saúde Bucal dos 293 municípios do Estado de Santa Catarina, Brasil, sobre o uso de fluoretos em saúde bucal coletiva. Métodos: estudo descritivo que investigou aspectos referentes às diversas formas de utilização de fluoretos e informações epidemiológicas de cárie dentária mediante questionários enviados a todos os 293 municípios do estado, no ano de 2005. Resultados: a taxa de resposta foi de 43,7\%; cerca de 60,0\% informaram sobre epidemiologia da cárie no município; apenas $1 / 4$ conhecia o percentual da população brasileira com acesso a água fluoretada; o custo da fluoretação foi ignorado por mais da metade dos profissionais $(61,7 \%)$; metade dos pesquisados revelou recomendar suplementos fluoretados para gestantes como método de prevenção de cárie; a indicação de fluoretos tópicos foi respondida corretamente por $63,3 \%$; mais de $80,0 \%$ revelaram conhecer as recomendações sobre bochechos e géis fluoretados, enquanto apenas $40,6 \%$ conheciam o uso do verniz. Conclusão: aspectos fundamentais sobre a utilização de fluoretos foram desconhecidos por parte considerável de coordenadores municipais, indicando necessidade de capacitação técnica sobre o tema.

Palavras-chave: flúor; prevenção; políticas públicas de saúde.

\begin{abstract}
Objective: the study aimed to evaluate the knowledge of municipal oral health authorities in the 293 municipalities of the State of Santa Catarina, Brazil, about use of fluoride in community dentistry. Methods: a descriptive study investigated questions regarding the use of different fluoride modalities and dental caries epidemiology through a self-completed pre-tested questionnaire, sent by courier, in 2005. Results: the response rate was 43.7\%; nearly $60.0 \%$ informed the existence of dental caries epidemiologic data in their cities; only $1 / 4$ showed knowledge about the percentage of Brazilian population benefit with water fluoridation access; the cost-effectiveness of fluoridation was unaware by more than a half of the respondents (61.7\%); half of the respondents recommended fluoride supplements for pregnant women; topical fluorides' indication were correctly answered by 63.3\%; more than $80.0 \%$ revealed knowledge about mouthrinses and gels, while only $40.6 \%$ knew about the use of varnishes. Conclusion: there is a considerable lack of knowledge about the utilization of fluorides among oral health municipal authorities, suggesting need of training on this topic.
\end{abstract}

Key words: fluoride; prevention; public bealth policies.

Endereço para correspondência:

Programa de Pós-Graduação em Epidemiologia, Universidade Federal de Pelotas, Av. Marechal Deodoro, 1160, $3 \circ$ Piso, Pelotas-RS, Brasil. CEP: $96020-220$

E-mail:andreiacascaes@hotmail.com 


\section{Introdução}

O flúor vem sendo utilizado em Saúde Pública desde meados do século XX como elemento eficaz, efetivo e seguro na prevenção e controle da cárie dentária. ${ }^{1}$ Existe uma variedade de formas de utilização dos fluoretos, por administração sistêmica e tópica, embora esteja bem estabelecido que sua principal ação preventiva é tópica. ${ }^{2-3}$

No Brasil, a água de abastecimento público é o principal veículo do flúor utilizado em escala populacional, por ser um método seguro, econômico e de grande alcance social. ${ }^{4}$ Juntamente com a fluoretação dos dentifrícios, massivamente utilizada a partir do final da década de 1980, a adição de flúor à água tem sido apontada como uma das principais razões da redução da prevalência de cárie na população brasileira. ${ }^{5-6}$

A utilização de bochechos e géis fluoretados alcançou popularidade em programas de saúde bucal coletiva no Brasil, especialmente em populações de risco à cárie com mais de seis anos de idade, devido ao bom custo-efetividade e facilidade de operacionalização, enquanto os vernizes fluoretados são recomendados para crianças em idade pré-escolar, ${ }^{7}$ sendo pouco empregados em programas de saúde bucal coletiva no país. ${ }^{4}$ A utilização de suplementos contendo flúor é contraindicada em gestantes, uma vez que não há fundamentação científica quanto ao mecanismo de ação do flúor, dose e benefícios para o bebê que justifiquem seu uso na gravidez. ${ }^{2}$

\section{No Brasil, a água de abastecimento público éo principal veículo do flúor utilizado em escala populacional, por ser um método seguro, econômico e de grande alcance social.}

Ações preventivas a base de fluoretos têm sido amplamente empregadas em programas de saúde bucal coletiva, embora sua adequação ao conhecimento científico atual não seja reconhecida. Recomendações ou guias de utilização de fluoretos em Saúde Pública são escassos no país, destacando-se o elaborado para o Estado de São Paulo. ${ }^{8}$ Apenas no ano de 2009, o Ministério da Saúde publicou o primeiro guia nacional de recomendações sobre o uso de fluoretos, tanto como métodos preventivos de âmbito populacional quanto para uso individual. ${ }^{4} 0$ propósito desse guia é dispor aos profissionais a possibilidade de opção pelo método ou associação de fluoretos mais adequado, a partir do diagnóstico da sua realidade locorregional.

Este trabalho foi realizado anteriormente à elaboração do guia nacional, quando não havia disponibilidade, nas secretarias de saúde dos municípios de Santa Catarina, de orientações padronizadas sobre a correta utilização dos fluoretos em saúde bucal coletiva. 0 conhecimento dos coordenadores municipais de saúde bucal sobre o uso dos fluoretos no cotidiano dos serviços de saúde, aqui investigado, e o fato de este estudo ser anterior à publicação do guia nacional devem contribuir com futuras análises, na avaliação da disseminação e utilização do material produzido pelo Ministério da Saúde.

Este estudo foi realizado com o propósito de investigar 0 grau de conhecimento sobre a utilização de fluoretos em Saúde Pública por parte dos coordenadores de saúde bucal dos municípios do Estado de Santa Catarina, Brasil, no ano de 2005.

\section{Métodos}

Tratou-se de um estudo descritivo que incluiu todos os 293 municípios do Estado de Santa Catarina (SC), Sul do Brasil. A população de Santa Catarina é estimada em 6.248.436 habitantes, sendo o décimo primeiro estado mais populoso do Brasil. ${ }^{9} 0$ Estado está dividido em nove macrorregiões: Extremo Oeste; Foz do Rio Itajaí; Grande Florianópolis (a Região Metropolitana da capital do Estado, Florianópolis-SC); Meio Oeste; Nordeste; Planalto Norte; Planalto Serrano; Sul; e Vale do Itajaí. 0 número de municípios e o percentual relativo da população de cada região em relação à população total são, respectivamente: $76(11,7 \%)$; $11(8,9 \%) ; 25(18,3 \%) ; 55$ (9,5\%); 13 (13,9\%); 13 $(5,7 \%) ; 18(4,6 \%) ; 43(14,5 \%)$; e $39(12,9)$.

Elaborou-se um questionário com perguntas sobre o conhecimento dos coordenadores municipais de saúde bucal acerca das diversas formas de utilização de fluoretos e dados epidemiológicos de cárie dentária no município. Algumas questões seguiram um padrão previamente utilizado, ${ }^{2}$ enquanto outras foram elaboradas especificamente para este estudo. 0 questionário foi pré-testado em alunos de Graduação e Pós-Graduação do curso de Odontologia da Universidade Federal de Santa Catarina, professores e profissionais da área de saúde bucal. 
0 questionário foi enviado - via postal - às Secretarias Municipais de Saúde dos 293 municípios do estado, no ano de 2005, com orientações para que 0 responsável pela área de odontologia/saúde bucal do município o respondesse. Na ausência do coordenador de odontologia/saúde bucal municipal, solicitou-se a um cirurgião-dentista, indicado pelo secretário municipal de saúde, que o respondesse. Para facilitar a resposta, anexos ao questionário, foram enviados envelopes previamente selados. Foram considerados como perdas os questionários não respondidos passado um mês da segunda postagem.

0 questionário foi estruturado em três blocos. 0 primeiro bloco de questões abordou os aspectos sociodemográficos e alusivos à formação do profissional participante, como sexo, idade, categoria profissional (cirurgião-dentista; outra categoria de nível superior; outra categoria de nível técnico), e tempo no exercício na coordenação. 0 segundo bloco referiu-se ao tipo de água mais frequentemente consumida no município, à fluoretação da água de abastecimento público, à existência e características de sistemas de vigilância sanitária dos teores de flúor (heterocontrole) e à existência de dados epidemiológicos de cárie dentária. 0 heterocontrole foi definido por Narvai como "o princípio segundo o qual, se um bem ou serviço qualquer implica risco ou representa fator de proteção para a saúde pública e então, além do controle do produtor sobre o processo de produção, distribuição e consumo, deve haver controle por parte das instituições do Estado". ${ }^{10}$ Recomenda-se que o heterocontrole da fluoretação das águas de abastecimento público seja operacionalizado por entidade ou instituição pública, privada ou de ambas índoles, diferente da companhia de abastecimento de água.

0 terceiro bloco apresentava questões referentes ao uso do flúor, como abrangência da fluoretação das águas no Brasil, concentração ótima recomendada de flúor nas águas e dentifrícios e custo da fluoretação das águas no Brasil. Adicionalmente, investigou-se o conhecimento dos coordenadores sobre o risco toxicológico do flúor, sobre a recomendação de suplementos fluoretados a gestantes e grupos de risco à cárie, e sobre a forma de utilização de géis, soluções para bochechos e vernizes fluoretados em programas de saúde bucal coletiva.

A definição das respostas corretas em relação à vigilância do flúor e fluoretação das águas de abas- tecimento público no Brasil foi baseada em artigos científicos acerca do tema. ${ }^{10,11}$ Para definir a resposta correta sobre o custo da fluoretação, considerou-se 0 intervalo entre $R \$ 1,00$ e $R \$ 1,50$, devido às diferentes fontes de informação. ${ }^{11,12}$ As respostas sobre métodos de aplicação tópica de flúor, utilização de fluoretos em programas preventivos, toxicologia do flúor e grupos de risco à cárie foram referenciadas a partir do guia de recomendações do Centers for Disease Control and Prevention/United States of America (CDC/USA) ${ }^{2}$ e de revisões sistemáticas da Cochrane Collaboration sobre o tema. ${ }^{13-16}$

Ao final do questionário, seguia um campo de dados, que deveria ser preenchido caso houvesse interesse por parte do participante em receber gratuitamente um CD-ROM contendo informações sobre a utilização das diversas formas de fluoretos, conforme publicação de Cury e colaboradores. ${ }^{17}$

Os dados obtidos foram digitados pelo programa Excel e, posteriormente, analisados pelo programa STATA 9.0. Realizou-se análise descritiva, obtendo-se as frequências relativas e absolutas das variáveis categóricas; e medidas de tendência central e dispersão, para variáveis quantitativas.

0 projeto de estudo, de $\mathrm{n}^{0} 203 / 04$, foi aprovado pelo Comitê de Ética em Pesquisa com Seres Humanos da Universidade Federal de Santa Catarina. Obteve-se 'Termo de Consentimento Livre e Esclarecido' de todos os participantes. As respostas fornecidas tiveram caráter sigiloso e voluntário, o nome de cada e todo participante não foi associado a qualquer uma das respostas dadas, as quais somente foram usadas para as finalidades expostas.

\section{Resultados}

Do total de 293 municípios de Santa Catarina, $128(43,7 \%)$ responderam à pesquisa. 0 número de municípios e a taxa de resposta, em ordem crescente para cada macrorregião, foi: $4(30,8 \%)$ para a região Nordeste; 4 (36,4\%) na região de Foz do Rio Itajaí; 5 $(38,5 \%)$ para o Planalto Serrano; 7 (38,9\%) para o Planalto Norte; 16 (41,0\%) para a região do Vale do Itajaí; 11 (44,0\%) para a Grande Florianópolis; 19 (44,2\%) para o Sul; 25 (45,5\%) para a região Meio Oeste; e 37 (48,7\%) para o Extremo Oeste. 0 somatório da população dos municípios respondentes representa 60,0\% da população do Estado de Santa Catarina. 
A média de idade dos coordenadores foi de 32,4 anos (desvio-padrão, $\mathrm{DP}=8,5$ ) e pouco mais da metade era do sexo feminino (52,3\%). 0 tempo médio de formado foi igual a 8,8 anos $(\mathrm{DP}=8,1)$ sendo que apenas um coordenador não apresentava formação universitária. 0 tempo médio de atividade de coordenação foi de 3,2 anos $(\mathrm{DP}=4,3)$.

0 tipo de água relatada como mais consumida nos municípios estudados $(70,3 \%)$ foi a água tratada pela companhia de abastecimento. Ao responder sobre a presença de flúor na água, $68,0 \%$ e 5,5\% dos coordenadores afirmaram apresentar em seus municípios, respectivamente, água artificialmente e naturalmente fluoretada. Entre os municípios que apresentavam água fluoretada artificialmente, 58,6\% afirmaram haver heterocontrole das águas (Tabela 1). Embora a presença de heterocontrole tenha sido confirmada em mais da metade dos municípios, cerca de $30 \%$ dos coordenadores relataram não saber qual a periodicidade da avaliação dos teores de flúor. A existência de dados epidemiológicos sobre a cárie dentária foi relatada pelos coordenadores de 78 municípios $(60,9 \%)$; entre estes coordenadores, a média - em anos - do último levantamento epidemiológico sobre cárie no município foi de 3,2 (DP=3,5). Apenas 67 coordenadores souberam informar o valor médio do número de dentes cariados, perdidos e restaurados (índice CPO-D) aos 12 anos de idade, obtido por ocasião do último levantamento epidemiológico. A média do CPO-D entre os respondentes foi de 3,8 (DP=1,8), com valores variando de 1,2 a 8,2 .

Observa-se (Figura 1) que mais da metade dos coordenadores $(55,5 \%)$ desconhecia qual o percentual da população brasileira com acesso a água de abastecimento público fluoretada. Quando o custo por habitante da fluoretação das águas de abastecimento público foi investigado, observou-se que 31,3\% dos coordenadores responderam corretamente (entre $\mathrm{R} \$ 1,00$ e $\mathrm{R} \$ 1,50)$. Mais da metade deles $(61,7 \%)$, entretanto, não soube precisá-lo e responder a essa

\section{Tabela 1 - Características dos municípios ( $n=128)$ segundo tipo predominante de água consumida pela população no Estado de Santa Catarina. Brasil, 2005}

\begin{tabular}{lcc}
\hline Variáveis & $\mathbf{n}$ & $\%$ \\
\hline Tipo de água consumida & & \\
$\quad$ Água tratada & 90 & 70,3 \\
Poços artesianos & 26 & 20,3 \\
Água mineral & 1 & 0,8 \\
$\quad$ Outra & 10 & 7,8 \\
$\quad$ Não sabe & 1 & 0,8 \\
Total & 128 & 100,0 \\
Presença de água fluoretada & & \\
$\quad$ Sim, fluoretada artificialmente & 87 & 68,0 \\
$\quad$ Não, fluoretada naturalmente & 7 & 5,5 \\
$\quad$ Não & 29 & 22,7 \\
$\quad$ Não sabe & 5 & 3,8 \\
Total & 128 & 100,0 \\
Existência de heterocontrole & & \\
$\quad$ Sim & & 58,6 \\
Não & 51 & 33,4 \\
$\quad$ Não sabe & 29 & 8,0 \\
Total & 07 & 100,0 \\
\hline
\end{tabular}

a) Considerados apenas os municípios que apresentavam fluoretação artificial das águas de abastecimento público. 


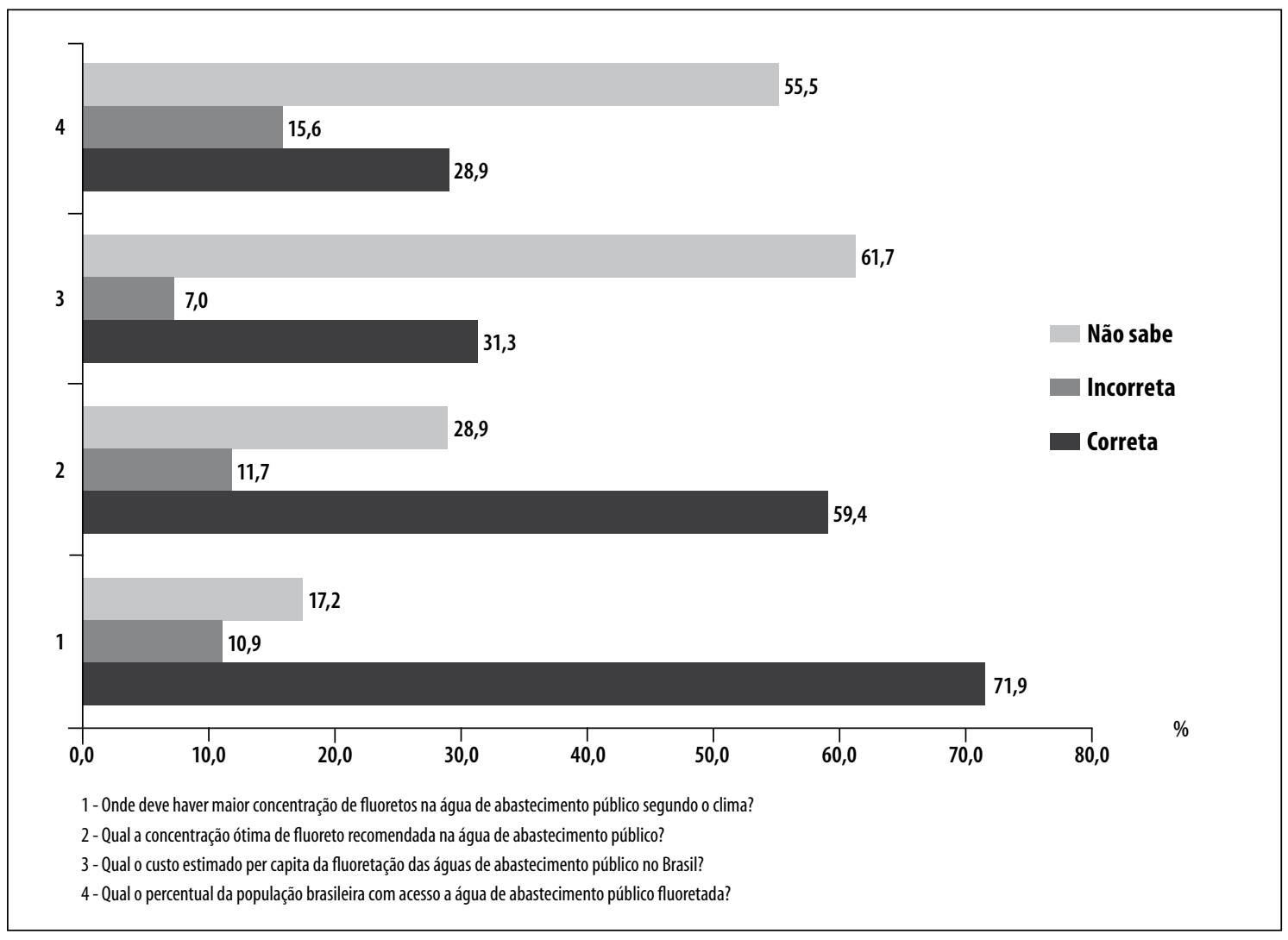

\section{Figura 1 - Distribuição dos municípios ( $n=128$ ) segundo conhecimento dos coordenadores municipais de Saúde Bucal sobre o uso do flúor no Estado de Santa Catarina. Brasil, 2005}

questão. 0 conhecimento sobre a concentração ótima de flúor recomendada na água de abastecimento público - entre 0,6 ppmF e 0,9 ppmF - foi observado em 59,4\% das respostas obtidas; porém, $28,9 \%$ dos coordenadores afirmaram não saber quais os níveis adequados de flúor. Quando foi abordada a influência das condições climáticas sobre a concentração de flúor na água de abastecimento público, a grande maioria dos coordenadores $(71,9 \%)$ respondeu, corretamente, que ela deve ser maior em cidades com climas mais frios.

A pergunta a respeito da faixa etária recomendada para utilização de bochechos com flúor em crianças revelou que grande parte dos coordenadores $(86,7 \%)$ detém a informação correta, da utilização do método em crianças com 6 anos ou mais. 0 mesmo foi observado quando se perguntou sobre a frequência recomendada - semanal - para a realização dos bochechos com fluoreto de sódio a $0,2 \%$ : um percentual de $88,3 \%$ dos coordenadores responderam corretamente. Não obstante, aproximados $30 \%$ de coordenadores desconhecia a frequência mínima de aplicação de gel flúor fosfato acidulado em programas de saúde bucal coletiva (Figura 2).

Sobre em que situações a associação de outro método tópico de fluoreto - além do dentifrício fluoretado - é recomendada, apenas 32,8\% dos coordenadores responderam corretamente: na ausência de flúor na água de abastecimento público.

0 conhecimento sobre os grupos de risco para a fluorose dentária foi corretamente apontado por $86,7 \%$ dos coordenadores e menos de $1,0 \%$ relatou desconhecer quais eram os grupos de risco (Figura 2).

Apesar de apenas 3,1\% terem afirmado desconhecer as situações para as quais se recomenda o uso de suplementos com flúor para gestantes, metade dos coordenadores não respondeu à questão corretamente: nunca existiu tal recomendação. 0 mesmo foi observado quando questionados sobre o uso de verniz 
fluoretado em programas de saúde bucal coletiva: uma pequena proporção $(4,7 \%)$ dos coordenadores não soube responder para que faixa etária é mais recomendado o uso de verniz, enquanto $40,6 \%$ responderam corretamente: em menores de 6 anos de idade. Resultado quase idêntico foi observado ao se questionar a frequência com que o verniz fluoretado deve ser utilizado: apenas 3,1\% não souberam respon- der e pouco menos da metade $(46,7 \%)$ respondeu corretamente - semestralmente (Figura 2).

\section{Discussão}

Os resultados encontrados no presente estudo apontaram o desconhecimento, por grande parte dos coordenadores participantes, de aspectos relevantes

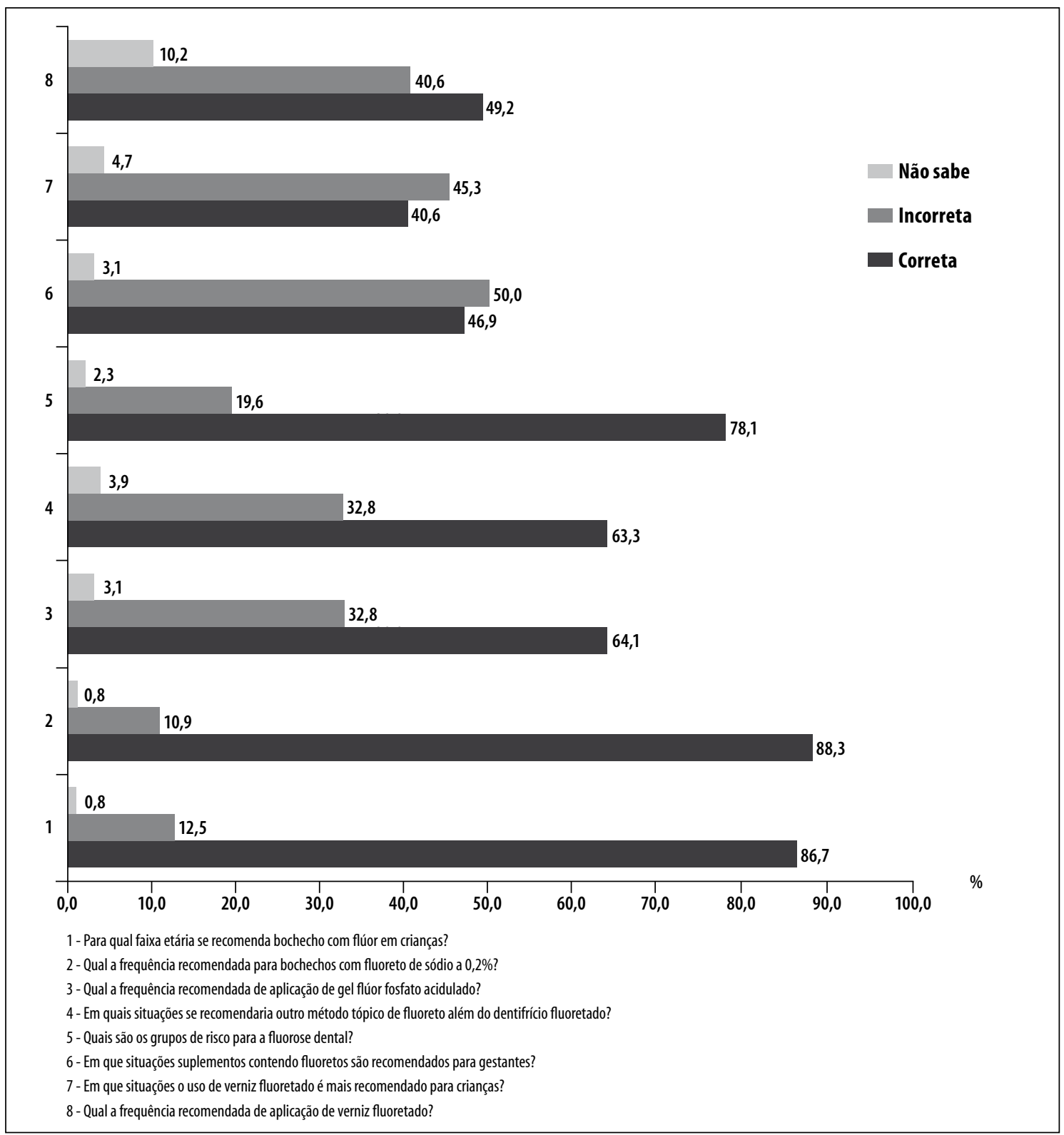

\section{Figura 2 - Distribuição dos municípios ( $n=128$ ) segundo conhecimento dos coordenadores municipais de Saúde Bucal a respeito da ação preventiva e/ou terapêutica do flúor sobre a cárie dentária em programas de saúde bucal coletiva no Estado de Santa Catarina. Brasil, 2005}


relacionados ao uso de fluoretos em Saúde Pública. Trata-se de um estudo original, desconhecem-se outros realizados com o mesmo objetivo. Se os resultados apresentados são capazes de contribuir para o desenho de ações e políticas específicas da área, a baixa taxa de resposta pode ser considerada uma limitação do trabalho em tela. Hipoteticamente, a ausência de resposta por parte dos que não concordaram em participar da pesquisa deve-se a seu menor grau de interesse ou maior dificuldade em responder às questões formuladas. Ainda assim, a taxa de resposta em estudos que utilizam o correio costuma ser baixa e os resultados deste artigo levam a conclusões otimistas, ${ }^{18}$ independentemente da estratégia adotada, ${ }^{19}$ o que evidencia a dificuldade em realizar estudos com uma metodologia pouco tradicional no Brasil.

A fluoretação das águas de abastecimento público é um método eficaz, seguro, de baixo custo e fácil aplicação. Segundo o Ministério da Saúde, sua continuidade e expansão devem ser estimuladas como elemento central das políticas de saúde bucal em todos os municípios brasileiros. ${ }^{4}$ Paralelamente à expansão da fluoretação, devem-se adotar sistemas de vigilância sanitária dos teores de flúor com base no princípio do heterocontrole, contribuindo, efetivamente, para melhorar a qualidade da fluoretação. ${ }^{20}$

Panizzi e Peres avaliaram o heterocontrole por período de dez anos, em município da região do Extremo Oeste de SC, revelando grande variação na concentração de flúor ao longo do tempo e nos diferentes pontos de coleta. ${ }^{21} \mathrm{~A}$ vigilância sanitária da fluoretação subsidiou intervenção político-jurídica da secretaria municipal de saúde, obtendo resultados efetivos (eram parciais, até então) no controle da adequação da fluoretação. ${ }^{21}$

Entre os coordenadores que afirmaram a existência de um sistema de heterocontrole no município, 30,0\% demonstraram não compreender a proposta desse sistema: ou seja, não souberam informar corretamente a frequência de monitoramento, ${ }^{10}$ nem a instituição que o realiza, apenas indicaram o responsável pela execução do heterocontrole, a estação de tratamento e abastecimento estadual que promove o controle operacional dos teores de flúor. 0 desconhecimento do funcionamento do sistema de heterocontrole implica possível ocorrência de super ou subdosagem da fluoretação das águas de abastecimento público, em alguns municípios de Santa Catarina. Subdosagens tornam a medida inócua, enquanto a superdosagem aumenta $o$ risco de fluorose dentária. ${ }^{20}$

0 fato de desconhecer a epidemiologia das doenças e os agravos bucais na população restringe o planejamento de ações efetivas de promoção da saúde bucal voltados às reais necessidades da população. A execução regular de levantamentos epidemiológicos de saúde bucal, recomendada pela Organização Mundial da Saúde - OMS -, pretende contribuir para a vigilância epidemiológica e 0 monitoramento e avaliação das medidas de intervenção preventivas e de controle da cárie. ${ }^{22}$ Aproximadamente metade dos coordenadores soube relatar o CPO-D médio do último levantamento epidemiológico de cárie realizado em seu município. Segundo dados do Levantamento Nacional de Saúde Bucal - SB-Brasil 2002/2003 -, o índice CPO-D da região Sul era de 2,3. ${ }^{23}$ Estudos transversais realizados no Estado de Santa Catarina apontam as seguintes médias de CPO-D aos 12 anos de idade: 1,4 em município da região da Grande Florianópolis; ${ }^{24}$ 1,91 e 1,84 em municípios da região Sul de SC; ${ }^{25}$ e 1,5 em município da região do Vale do Itajaí. ${ }^{26}$

Em relação ao percentual da população brasileira com acesso a água fluoretada, apenas $1 / 4$ dos coordenadores soube se aproximar da proporção correta, em torno dos $50,0 \%$ quando da realização da pesquisa. Entende-se que o acesso a água tratada e fluoretada é fundamental para as condições de saúde da população. A viabilização de políticas públicas que garantam a implantação da fluoretação das águas e a ampliação dos municípios com sistemas de tratamento é a forma mais abrangente - e socialmente justa - de acesso ao flúor. ${ }^{27} \mathrm{O}$ desenvolvimento de ações intersetoriais de ampliação da fluoretação das águas no Brasil também foi atribuído ao conhecimento da importância dessa medida e sua popularização.

Quanto ao custo da fluoretação das águas de abastecimento público no Brasil, a maioria dos participantes desconhecia ou não soube informar corretamente seu valor (em Reais). Esse custo social, economicamente suportável, de $R \$ 1,00$ a $R \$ 1,50$ pessoa/ano, torna-se uma das variáveis atuantes na transformação significativa do perfil epidemiológico da cárie, além de diminuir a desigualdade social no acesso a um produto fluorado, beneficiando, indistintamente, todos os estratos da população. ${ }^{12}$ É um valor que varia de acordo com o tamanho da população do município, número de pontos de fluoretação da água de abastecimento, quantidade e tipo de equipamentos de monitoramento utilizados, 
quantidade e custo dos produtos químicos fluorados, custo de transporte, entre outros aspectos. ${ }^{2,12}$

Ao abordar a concentração ótima de flúor na água de abastecimento público, pouco mais da metade dos pesquisados respondeu corretamente à questão. A concentração ótima de flúor na água de abastecimento público no Brasil varia de $0,6 \mathrm{ppmF}$ a $0,9 \mathrm{ppmF}^{28}$ a depender da média de temperatura da área. Populações de lugares com clima mais quente necessitam menor concentração de fluoretos na água - que consomem em maior quantidade -, ao contrário dos habitantes de regiões com temperaturas médias mais baixas. ${ }^{29}$

Cumpre destacar que mais da metade dos coordenadores pesquisados indicou o uso de suplementos fluoretados para gestantes em alguma situação, quando não há justificativa científica que recomende seu uso por essa população. ${ }^{2}$ Evidências apontam ausência de diferenças significativas na redução de cárie de crianças cujas mães ingeriram flúor durante a gestação, comparativamente ao grupo-controle. ${ }^{30}$ Suplementos de flúor, como medicamentos, são de prescrição individual, sem efetividade como medida de Saúde Pública.

Apesar das recomendações sobre o uso de fluoretos em programas preventivos terem sido respondidas corretamente pela maioria dos pesquisados, os métodos de utilização dos vernizes e géis são menos conhecidos do que $o$ bochecho fluoretado. A utilização de bochechos em programas preventivos surgiu nas décadas de 1960 e 1970, nos países escandinavos e nos Estados Unidos da América, alcançando grande popularidade como medida de saúde bucal coletiva. Este estudo confirmou que a grande maioria dos participantes recomenda o uso de bochechos com $0,2 \%$ de fluoreto de sódio para crianças com idade igual ou maior que 6 anos, uma vez por semana.

As formas cientificamente embasadas de utilização de gel fluoretado não são tão conhecidas como os bochechos. Mais de 30,0\% dos respondentes demonstrou desconhecer a frequência mínima recomendada para obtenção efetiva de redução de cárie, o que pode influenciar na tomada de decisão relacionada ao uso de fluoretos. Parece que a utilização de géis fluoretados enquanto agente remineralizador - na prática clínica individual - e como medida preventiva de caráter populacional não está bem conhecida entre os coordenadores pesquisados. É importante destacar que o gel tem alta concentração de fluoretos e, se utilizado constantemente, aumenta o risco de ingestão e, consequentemente, de fluorose dentária. Sabe-se, igualmente, que a múltipla exposição aos fluoretos aumenta o risco de fluorose. ${ }^{4}$

Baseado em revisões sistemáticas da Cochrane Collaboration, o uso semanal regular de bochechos fluoretados está claramente associado à efetiva redução de cárie, em até $26,0 \% \cdot{ }^{13} 0$ gel flúor fosfato acidulado, se aplicado duas vezes ao ano, tem potencial de reduzir a experiência da cárie em $21,0 \% .^{15}$ Porém, crianças menores de 6 anos de idade, de maneira geral, não deveriam realizar bochechos e géis fluoretados, haja vista não apresentarem reflexo motor de controle da ingestão, o que aumentaria o risco para fluorose dentária. ${ }^{2}$

Outro método tópico é o verniz fluoretado, efetivo na redução da cárie em até $46,0 \%$ em dentes permanentes e em 33,0\% em dentes decíduos, quando aplicado com frequência semestral: ${ }^{16}$ sua consistência pegajosa, por mais tempo em contato com o esmalte dentário, torna-o mais indicado para menores de 6 anos de idade. ${ }^{2}$ Menos da metade dos coordenadores pesquisados informou corretamente a idade $(40,6 \%)$ e frequência $(46,7 \%)$ mais recomendada para o uso desse método.

Como tudo na natureza, o flúor também pode ser tóxico. Sua toxicidade pode-se caracterizar como aguda ou crônica: aguda, quando relacionada à ingestão de grande quantidade de flúor de uma única vez; e crônica pela ingestão de pequenas, porém excessivas quantidades de flúor, por período prolongado. ${ }^{17} \mathrm{~A}$ 'dose provavelmente tóxica' (DPT) para a ocorrência de manifestação aguda da intoxicação por flúor é de $5 \mathrm{mgF} / \mathrm{kg}$, enquanto uma dose de $0,05-0,07 \mathrm{mgF} / \mathrm{kg} /$ dia tem sido sugerida como limite máximo de risco para a fluorose dentária, manifestação crônica da intoxicação por flúor. ${ }^{17}$

Crianças menores de 9 anos de idade que usam dentifrício fluoretado em excesso - frequência maior que três vezes ao dia -, sem supervisão dos pais ou responsáveis, estão incluídas no grupo de risco para fluorose dentária. ${ }^{2}$ No presente estudo, $86,7 \%$ dos coordenadores identificou corretamente esse grupo de risco. 0 guia de recomendações brasileiro recomenda que crianças menores de 9 anos utilizem pequenas quantidades de pasta (0,3 grama, correspondente a um grão de arroz) como forma de reduzir o risco para fluorose. ${ }^{4}$ Portanto, uma frequência de escovação de duas vezes ao dia, com quantidade suficiente de dentifrício fluoretado, diminui a possível ingestão de flúor e reduz a cárie em até $24,0 \%{ }^{14}$ 
Em saúde bucal coletiva, a indicação do uso dos fluoretos deve estar de acordo com o risco à cárie da população beneficiada. Grupos com baixo risco à cárie podem manter essa condição apenas com a frequente exposição à água de abastecimento fluoretada, em níveis adequados e acompanhada do uso de dentifrícios fluoretados. ${ }^{2} \mathrm{O}$ uso de fluoretos tópicos em população de baixo risco à cárie não adiciona efeito preventivo, além de aumentar o risco para fluorose. ${ }^{31}$ Grupos e indivíduos considerados de alto risco, entretanto, deveriam ser beneficiados com a exposição adicional de fluoretos, como bochechos, géis ou vernizes. Grupos de maior risco à cárie dentária incluem: a) expostos a água de abastecimento sem flúor; b) expostos a água de abastecimento com teores de fluoretos abaixo da concentração indicada (até 0,54 ppmF); c) cujo CPOD médio (aos 12 anos de idade) seja maior do que $3 ;$ d) em que menos de 30,0\% de seus indivíduos do grupo sejam livres de cárie aos 12 anos de idade; e e) residentes em áreas de pobreza, onde se estima serem menores os níveis de exposição geral aos fluoretos. ${ }^{4}$ Cerca de 1/3 dos coordenadores pesquisados afirmou - corretamente - que métodos tópicos de aplicação coletiva, além de dentifrício fluoretado, são indicados para populações de alto risco à cárie. 0 uso adicional de fluoretos tópicos só é recomendado para popula-

\section{Referências}

1. Centers for Disease Control and Prevention. Ten great public health achievements: United States, 19001999. Morbidity and Mortality Weekly Report. 1999; 48(12):241-243.

2. Centers for Disease Control and Prevention. Recommendations for using fluoride to prevent and control dental caries in United States. Morbidity and Mortality Weekly Report. 2001; 50(RR14):1-42.

3. Tenuta LM, Cury JA. Fluoride: its role in dentistry. Brazilian Oral Research. 2010; 24 Suppl 1:9-17.

4. Ministério da Saúde. Secretaria de Atenção à Saúde. Departamento de AtençãoBásica. Guia para recomendações do uso de fluoretos no Brasil. Brasília: Ministério da Saúde; 2009.

5. Cury JA, Tenuta LMA, Ribeiro CCC, Paes Leme AF. The importance of fluoride dentifrices to the current dental caries prevalence in Brazil. Brazilian Dental Journal. 2004; 15(3):167-174. ções consideradas de alto risco à cárie, alcançando importante efeito preventivo. ${ }^{3}$

0 uso correto de fluoretos reduz a prevalência, incidência e gravidade da cárie dentária e suas sequelas, de maneira segura e efetiva, com ótima relação custo/ benefício. Recomendações oficiais acerca do uso de fluoretos em saúde bucal coletiva são desejáveis e deveriam ser amplamente adotadas.

0 presente estudo concluiu que aspectos fundamentais relacionados ao uso de fluoretos em saúde bucal coletiva eram desconhecidos por parte considerável dos coordenadores de saúde bucal participantes da pesquisa. Essa é a principal razão porque seus autores consideram necessária a capacitação técnica do conjunto de coordenadores municipais de saúde bucal do Estado de Santa Catarina na utilização de fluoretos em saúde bucal coletiva.

\section{Contribuição dos autores}

Cascaes AM planejou o estudo, participou da coleta e análise de dados e redigiu o manuscrito.

Kamimura LCB planejou o estudo, participou da coleta e análise de dados e colaborou na redação do artigo.

Peres KG e Peres MA orientaram o planejamento do estudo, participaram da análise dos dados e realizaram a revisão crítica do manuscrito.

6. Ramires I, Buzalaf MA. A fluoretação da água de abastecimento público e seus benefícios no controle da cárie dentária - cinquenta anos no Brasil. Ciência e Saúde Coletiva. 2007; 12(4):1057-1065.

7. Carvalho DM, Salazar M, Oliveira BH, Coutinho ES. Fluoride varnishes and decrease in caries incidence in preschool children: a systematic review. Revista Brasileira de Epidemiologia. 2010; 13(1):139-149.

8. Narvai PC, Forni TIB, Junqueira RS, Cury JA, Castellanos RA, Soares MC. Uso de produtos fluorados conforme o risco de cárie dentária: uma revisão crítica. Revista da Associação Paulista dos CirurgiõesDentistas. 2002; 56(2):101-107.

9. Instituto Brasileiro de Geografia e Estatística. Censo brasileiro de 2010. Rio de Janeiro: Instituto Brasileiro de Geografia e Estatística; 2010.

10. Narvai PC. Fluoretação da água: heterocontrole no município de São Paulo no período 1990-1999. 
Revista Brasileira de Odontologia em Saúde Coletiva. 2000; $1(2): 50-56$.

11. Ministério da Saúde. Área técnica de saúde bucal: fluoretação da água de consumo público: parecer técnico científico de saúde bucal. Brasília: Ministério da Saúde; 1996.

12. Frias AC, Narvai PC, Araújo ME, Zilbovicius C, Antunes JLF. Custo da fluoretação das águas de abastecimento público, estudo de caso - Município de São Paulo, Brasil, período de 1985-2003. Cadernos de Saúde Pública. 2006; 22(6):1237-1246.

13. Marinho VC, Higgins JP, Logan S, Sheiham A. Fluoride mouthrinses for preventing dental caries in children and adolescents. Cochrane Database Systematic Review. 2003; (3):CD002284.

14. Marinho VC, Higgins JP, Sheiham A, Logan S. Fluoride toothpastes for preventing dental caries in children and adolescents. Cochrane Database Systematic Review. 2003; (1):CD002278.

15. Marinho VC, Higgins JP, Logan S, Sheiham A. Fluoride gels for preventing dental caries in children and adolescents. Cochrane Database Systematic Review. 2002; (2):CD002280.

16. Marinho VC, Higgins JP, Logan S, Sheiham A. Fluoride varnishes for preventing dental caries in children and adolescents. Cochrane Database Systematic Review. 2002; (3):CD002279.

17. Cury JA. Uso do flúor e controle da doença cárie. In: Baratieri LN, Monteiro S, Andrada MAC, Vieira LCC, Ritter AV, Cardoso AC. Odontologia Restauradora Fundamentos e Possibilidades. São Paulo: Santos; 2001. p. 33-68.

18. Acurcio FA, Rozenfeld S, Ribeiro AQ, Klein CH, Moura CS, Andrade CR. Utilizacao de medicamentos por aposentados brasileiros: 1--metodologia e resultados de cobertura de inquérito multicêntrico. Cadernos de Saúde Pública. 2006; 22(1):87-96.

19. Gouveia VV. Taxa de resposta em levantamento de dados pelo correio: o efeito de quatro variáveis Psicologia: Teoria e Pesquisa. 1995; 11(2):163-168.

20. Schneider Filho DA, Prado IT, Narvai PC, Barbosa SR. Fluoretação da água: como fazer a vigilância? Rio de Janeiro: CEDROS; 1992. (Caderno de Saúde Bucal; $\left.\mathrm{n}^{0} 2\right)$.
21. Panizzi M, Peres MA. Dez anos de heterocontrole da fluoretação de águas em Chapecó, Estado de Santa Catarina, Brasil. Cadernos de Saúde Pública. 2008; 24(9):2021-2031.

22. Organização Mundial da Saúde. Levantamentos básicos em Saúde Bucal. 4ª São Paulo: Santos; 1999.

23. Ministério da Saúde. Relatório Projeto SB Brasil 2003: condições de saúde bucal da população brasileira 2002-2003: resultados principais. Brasília: Ministério da Saúde; 2004.

24. Bastos JL, Nomura LH, Peres MA. Tendência de cárie dentária em escolares de 12 e 13 anos de idade de uma mesma escola no período de 1971 a 2002, em Florianópolis, Santa Catarina, Brasil. Cadernos de Saúde Pública. 2004; 20(1):117-122.

25. Traebert J, Suarez CS, Onofri DA, Marcenes W. Prevalência e severidade de cárie dentária e necessidade de tratamento odontológico em pequenos municípios brasileiros. Cadernos de Saúde Pública. 2002; 18(3):817-821.

26. Traebert JL, Peres MA, Galesso ER, Zabot NE, Marcenes W. Prevalência e severidade da cárie dentária em escolares de seis e doze anos de idade. Revista de Saúde Pública. 2001; 35(3):283-288.

27. Ministério da Saúde. Diretrizes da Política Nacional de Saúde Bucal. Brasília: Ministério da Saúde; 2004.

28. Frazao P, Peres MA, Cury JA. Qualidade da água para consumo humano e concentração de fluoreto. Revista de Saúde Pública. No prelo 2011.

29. Heller KE, Sohn W, Burt BA, Eklund SA. Water consumption the United States in 1994-1996 and implications for water fluoridation policy. Journal of Public Health Dentistry. 1999; 59(1):3-11.

30. Leverett DH, Adair SM, Vaughan BW, Proskin HM, Moss ME. Randomized clinical trial of the effect of prenatal fluoride supplements in preventing dental caries. Caries Research. 1997; 31(3):174-179.

31. Iwakura ML, Morita MC. Prevenção de cárie dentária por bochechos com flúor em município com água fluoretada. Revista Panamericana de Salud Publica. 2004; 15(4):256-261.

Recebido em 28/02/2011

Aprovado em 13/03/2012 